DOI: $10.24850 /$ j-tyca-2018-06-05

Artículo

\title{
Propuesta del modelo físico del infiltrómetro de cilindros concéntricos rediseñado multifuncional (ICCRM)
}

\section{Proposal of the physical model of the multifunctional redesigned double ring infiltrometer (ICCRM)}

Eduardo Teófilo-Salvador ${ }^{1}$

Guillermo Pedro Morales-Reyes ${ }^{2}$

${ }^{1}$ Centro Interamericano de Recursos del Agua de la Universidad Autónoma del Estado de México, mca.ts.eduardo2015@gmail.com

${ }^{2}$ Centro Interamericano de Recursos del Agua de la Universidad Autónoma del Estado de México, gpmoralesr@uaemex.mx

Autor para correspondencia: Eduardo Teófilo-Salvador, mca.ts.eduardo2015@gmail.com

\section{Resumen}

Debido a la necesidad de mejorar las técnicas y métodos de medición de infiltración de agua en el suelo, diversos autores han propuesto modificaciones a equipos comúnmente utilizados, tal como el infiltrómetro de cilindros concéntricos. El objetivo esta investigación fue revisar el funcionamiento del infiltrómetro de cilindros concéntricos tradicional, y a partir de ello, diseñar, construir y verificar de manera funcional, un modelo físico de infiltrómetro con las características necesarias para generar procesos de saturación y sobresaturación en el suelo. Se detectaron problemas para la obtención de los valores iniciales de infiltración a partir de la revisión del funcionamiento tradicional; atendiendo esta situación se presentó el diseño del infiltrómetro multifuncional acoplado a los cilindros concéntricos. Posteriormente se adquirieron y construyeron las piezas necesarias para el nuevo modelo, y se verificó la funcionalidad para realizar los ajustes necesarios. Por 
últmo, se comparó in situ con el equipo tradicional. Los resultados obtenidos mostraron que el infiltrómetro de cilindros concéntricos rediseñado multifuncional permite controlar una carga hidráulica de inundación constante desde $50 \mathrm{~mm}$, hasta $300 \mathrm{~mm}$, o bien, una carga variable, mixta o combinada; también es posible mantener la superficie exterior húmeda, además de controlar el escurrimiento superficial gracias a los dispositivos de control. Con el nuevo modelo físico de infiltrómetro se satura y sobresatura una masa de suelo a partir de reproducción a escala reducida de eventos, tales como las precipitaciones según la intensidad, duración y frecuencia de incidencia, con ello el estudio de la distribución de flujo de agua en el suelo.

Palabras clave: infiltrómetro multifuncional, carga hidráulica, saturación, sobresaturación.

\begin{abstract}
Due to the need to improve the technique and methods of measurement of soil water infiltration, several authors have proposed modifications to commonly used equipment, such as the double ring infiltrometer. The objective of the research was to review the operation of the traditional double ring infiltrometer and from there to design, construct and functionally verify a physical model of infiltrometer with the necessary characteristics to generate processes of saturation and supersaturation in the soil. From the review of the traditional operation problems were detected for obtaining of the initial infiltration values, attending this situation was presented the design of the multifunctional infiltrometer coupled to the concentric cylinders. Later were acquired and built the necessary parts for the new model, and verified the functionality to make the necessary adjustments, finally it was compared in situ with the traditional equipment. As results it was obtained that, the multifunctional redesigned double ring infiltrometer allows control a constant hydraulic flood load from $50 \mathrm{~mm}$ to $300 \mathrm{~mm}$, or variable load mixed or combined, it is also possible to maintain the wet outer surface, besides controlling the surface runoff, thanks to the control devices. With the new physical model of infiltrometer it become saturated and supersaturated a mass of soil from a reproduction reduced scale of winds such as rainfall according to the intensity, duration and frequency of incidence, with it the study of the distribution water flow in the soil.
\end{abstract}

Keywords: multifunctional infiltrometer, hydraulic load, saturation, supersaturation. 
Recibido: 05/07/2017

Aceptado: $17 / 04 / 2018$

\section{Introducción}

Actualmente, se han desarrollado diversas técnicas y equipos para medir la infiltración de agua en el suelo, así como también para predecir y simular el comportamiento bajo supuestos empíricos, analíticos o numéricos. Aunque la mayoría de los equipos existentes son de aplicación experimental y de investigación, el desarrollo de nuevas aplicaciones para estos equipos no ha sido notable; tal como el infiltrómetro de cilindros concéntricos, del cual existen reportes de mejoras adicionales (como las de sus dimensiones), así como para mantener cargas de inundación constantes automatizadas.

Touma y Albergel (1992), indican que el rango de mediciones del infiltrómetro de cilindros concéntricos es más amplio que las de un simulador de lluvia, debido a los bajos costos de adquisición, instalación y operación. Además de que este equipo permite determinar la velocidad de infiltración, la infiltración acumulada (Bouwer, 1986), y la conductividad hidráulica saturada (Álvarez, Carral, Hernández \& Almendros, 2013; Reynolds \& Reddy, 2012; Neris, Jiménez, Fuentes, Morillas \& Tejedor 2012; Ronayne, Houghton, \& Stednick, 2012; Fodor, Sándor, Orfanus, Lichner \& Rajkai, 2011; Köhne et al., 2011; ZapataSierra \& Manzano-Agugliaro, 2008; Sharratt, Zhang \& Sparrow, 2006).

Este infiltrómetro es muy utilizado a pesar de la variación en sus dimensiones, es por ello que Pinheiro, Poeta y Kaufmann, (2009) utilizaron cilindros de 25 y $50 \mathrm{~cm}$ de diámetro y $30 \mathrm{~cm}$ de altura. Ahmed, Osman y Ibrahim (2012) construyeron un juego de 23 y $75 \mathrm{~cm}$ de diámetro y $15.3 \mathrm{~cm}$ de altura. El-Hames y Al-Wagdany (2013) aumentaron las dimensiones a $2.5 \mathrm{~m}$ de diámetro. Pero dichas dimensiones tienen efectos, según Ahuja, El-Swaify y Rahman (1976), demuestran que, cuando el cilindro exterior es de $90 \mathrm{~cm}$ de diámetro y el interior de $30 \mathrm{~cm}$, el flujo lateral es eliminado. Bouwer (1986) sugirió que el diámetro de los cilindros al menos debe de ser de $100 \mathrm{~cm}$ para 
medir la conductividad hidráulica saturada. La American Society for Testing and Materials (1994) considera que el agua de infiltración del cilindro exterior provoca la presencia del flujo vertical debajo del cilindro interior. Lai, Luo y Ren, (2010) concluyeron que para obtener la conductividad hidráulica saturada el cilindro interior debe tener un diámetro mayor de $80 \mathrm{~cm}$. Por su parte, Teófilo (2010) obtuvo que la tasa de infiltración lateral es mucho mayor que la tasa de infiltración vertical para diferentes suelos superficiales, aun con diámetro interior y exterior de $31 \mathrm{~cm}$ y $46 \mathrm{~cm}$ respectivamente, y altura de $45 \mathrm{~cm}$.

Del mismo modo que en la aplicación del infiltrómetro también influyen diversos factores, tal que Fatehnia y Tawfiq (2014) consideraron el efecto del encharcamiento, profundad del cilindro en el suelo, la saturación efectiva inicial y el tipo de suelo. Pero también un buen conocimiento del funcionamiento del equipo permite relacionar sus mediciones a otros estudios, como el de Chin (2008); Chen y Wuing (2002), y Zhang, Peng, Wang, Zhao, \& Lin (2013), quienes asociaron la capacidad de infiltración a una relación precipitación-recarga del agua subterránea a diferentes profundidades. Lichner, Eldridge, Schacht, Zhukova, Holko, Sir y Pecho (2011), y Jialilang, Bo, Tao, Xunquiang, Moerong y Henry (2012) estimaron la infiltración efectiva relacionada con la percolación en el subsuelo con este equipo. Andrade y Muralidharan (2011) estimaron la tasa de infiltración asociada a la percolación en un suelo con pendiente topográfica. Raoof, Hosein, Ashraf, \& Marofi (2011) relacionaron el incremento de la tensión capilar con la inclinación de la pendiente topográfica usando el infiltrómetro. Por su parte, Teófilo (2015) determinó la infiltración eficaz inicial en el límite superior de la zona no saturada donde las tasas de infiltración fueron muy similares, debido a la similitud de la presión que ejerce el suelo en el eje $x, y$ y $z$. De igual forma, se han realizado estudios de coeficientes de dispersión utilizando propuestas de tripe anillo (Zhang, Qi, Zhou, \& Pang 2006).

Cabe destacar que, para una recolección de datos adecuada y control del equipo se han realizado mejoras, de esta forma Constantz y Murphy (1987) utilizaron un transductor de presión, con el que desarrollaron un depósito tipo Mariotte automatizado, con el objetivo de mantener el flujo de carga constante. Por su parte, Matula y Dirksen (1989) implementaron un sistema semiautomático para carga constante con regulación de $\pm 1 \mathrm{ml}$. Maheshwari (1996) referido por Milla y Kish (2006) diseñó un infiltrómetro automatizado con sensores de nivel, de capacitancia, válvulas senoidales y baterías. Gregory, Dukes, Miller y Jones (2005) incluyeron un sifón Mariotte para carga constante en el 
cilindro interior. Milla y Kish (2006) emplearon un sistema de sensores de medición del nivel de agua en infrarrojo, usados para carga descendente y carga constante, con apoyo del depósito Mariotte. Lazarovitch, Ben-Gal, Šimůnek y Shani (2007) dispusieron de un infiltrómetro de un solo cilindro semiautomático de carga constante. Arriaga, Kornecki, Balkcom y Raper (2010) desarrollaron un infiltrómetro de cilindros concéntrico simple automatizado, con condiciones de descenso de carga hidráulica. Ong, Werkema y Lane (2012) usaron una combinación de transductores de presión, microcontroladores y fuentes electrónicas abiertas, creando un sistema, tanto para carga constante como para descendente. Fathehina et al. (2016) incluyeron microcontroladores, bomba, sensores de nivel y válvulas de flotación a nivel constante.

Aunque se han desarrollado sistemas de control automatizados, no existe variación significativa entre obtener mediciones manuales y automatizadas (Gómez-Tagle, Gutiérrez \& Zepeda, 2010), como las reportadas para la infiltración acumulada, por lo que el ahorro en tiempo es proporcional al costo de un equipo automatizado (Salas, 2012). Asimismo, la evolución no ha cambiado radicalmente, más que en automatizar y mantener carga constante o carga descendente dentro de los cilindros.

\section{Objetivo}

El objetivo de la investigación fue revisar el funcionamiento del infiltrómetro de cilindros concéntricos tradicional y a partir de ello, diseñar, construir y verificar funcionalmente un modelo físico de infiltrómetro, con las características necesarias para generar procesos de saturación y sobresaturación en el suelo.

\section{Materiales y métodos}

\section{Revisión del infiltrómetro de cilindros concéntricos tradicional}


Se realizó una revisión bibliográfica del uso y funcionamiento del infiltrómetro, de la cual se encontró que la esencia de la metodología tradicional del infiltrómetro de cilindros concéntricos no ha cambiado notablemente; por ejemplo, el llenado de los cilindros se ha modificado poco, y éste puede ser mediante vaciado directo de agua, o bien, colocando una fuente de suministro (manguera) en un solo punto, pero de ello, el agua comienza a infiltrar antes de alcanzar el nivel deseado dentro de los cilindros en un tiempo de 2 a 3 minutos; además se genera un lavado de la superficie por la turbulencia ocasionada, la variación de niveles en ambos cilindros existe cuando no se llenan de manera simultánea (Teófilo, 2010; Pinheiro et al., 2009), lo que genera flujos preferenciales, los cuales se reflejan en una diferencia en la tasa de infiltración (Chen et al., 2002; Návar y Synnott, 2000), además de que al incrementar el diámetro y la carga hidráulica de inundación, aumenta la longitud de la zona húmeda y se reduce la infiltración acumulada al incrementar la profundidad de prueba (Chowdary, Damodhara \& Jaiswal, 2006).

\section{Propuesta de diseño}

En esta etapa primero se idealizó el funcionamiento más adecuado para generar procesos de saturación y sobresaturación de una masa de suelo, utilizando el infiltrómetro de cilindros concéntricos. Se consideró que para saturar una capa superior de suelo de $40 \mathrm{~cm}$ son necesarios de 20 a $50 \mathrm{~mm} / \mathrm{h}$ (El-Hames et al., 2013) o con una carga constante de 50 $\mathrm{mm}$ sobre la superficie del suelo (Ahmed et al., 2012), cuyo valor sería el mínimo requerido para carga de inundación constante dentro de los cilindros.

De acuerdo con lo anterior, se pensó en un mecanismo dentro de los cilindros que permitiera controlar una columna de agua a diversas alturas y que éste cerrara el paso de agua al alcanzar la lámina deseada. Además de que en la superficie de contacto, la distribución de agua fuese uniforme para eliminar la turbulencia y cambios de presión por flujos de compensación, por lo que un sistema de aspersión mejoraría los resultados en un $33 \%$ con respecto a la metodología tradicional (Teófilo, 2015). 
Con las bases anteriores se propuso un modelo que consistió en un sistema de suministro, distribución y operación del infiltrómetro de cilindros concéntricos rediseñado multifuncional (ICCRM), es decir, desde una fuente de suministro de agua, hasta el control de la carga de agua dentro de los cilindros. Este modelo se constituye en tres subsistemas, los cuales se detallan a continuación:

Subsistema 1. Infiltrómetro de cilindros concéntricos rediseñado multifuncional. Nombrado así, debido a las funciones que se obtienen gracias a las partes que lo constituyen en la nueva propuesta, mostradas en la Figura 1 y que se describen a continuación:

a) Conjunto de aspersores circulares. Para las áreas de contacto del agua con el suelo se propusieron aspersores circulares con manguera de diámetro de $1.27 \mathrm{~cm}$, éstas con dos derivaciones de alimentación del mismo material en cada conjunto, y para el área el exterior se consideró una ramificación en curva de tipo $Y$ ondulada de CPVC (policloruro de vinilo clorado). Bajo criterio, en los aspersores se proyectaron perforaciones de diámetro $0.16 \mathrm{~cm}$, cada $5 \mathrm{~cm}$ de separación en el eje medio lateral y transversal hacia abajo.

b) Operación de carga fija. Para mantener una carga de diseño constante mínima de $50 \mathrm{~mm}$, fue necesario considerar válvulas con flotador sumergibles de dimensiones pequeñas, especialmente de ancho no mayor a $7 \mathrm{~cm}$. Se pensaron dos flotadores, uno interior y otro intermedio (entre los dos cilindros). Para el caso exterior se planteó una válvula con flotador no necesariamente sumergible. Asimismo, se tomó en cuenta que estos dispositivos contaran con una válvula de paso para el control de la velocidad de salida y manguera derivadora tipo sanitario, para enviar y regular el caudal de agua hacia las derivaciones de alimentación.

c) Conductos dobles de alimentación mixta. Para cada cilindro, se consideró alimentar a los aspersores circulares y a la válvula con flotador de forma independiente con tubo de CPVC de diámetro $1.90 \mathrm{~cm}$ graduados longitudinalmente. En el tubo se adaptó una miniválvula de abertura y cierre para acoplar la manguera derivadora de la válvula con flotador, como se observa en la Figura 1. Estas miniválvulas también sirven para manipular el vertido por aspersión en toda el área de contacto o por inundación en un solo punto. Para el caso exterior se propusieron dos tubos, uno de $1.90 \mathrm{~cm}$ y otro de $1.27 \mathrm{~cm}$, en el extremo del primer tubo se adhieren con codos a la válvula con flotador y de éste sale un tramo de manguera de $1.27 \mathrm{~cm}$ unido al aspersor correspondiente, con la finalidad de controlar el humedecimiento fuera 
de los cilindros. El tubo de $1.27 \mathrm{~cm}$ alimenta de manera directa al aspersor circular exterior.

d) Conductos de alimentación variable. Este conjunto tiene como objetivo regular el caudal de alimentación mediante flujómetros en caso de requerir cargas variables. Para el control del vertido de agua en el suelo, se propuso colocar una derivación tipo $\mathrm{Y}$ de la salida del flujómetro de diámetro de $1.27 \mathrm{~cm}$, para adaptar los conductos dobles de alimentación mixta con codos de $90^{\circ}$ de diámetro, $1.27 \mathrm{~cm}$ a 1.90 $\mathrm{cm}$. En la derivación tipo $\mathrm{Y}$ se consideró que tuvieran válvulas tipo esfera para desviar el agua a cualquier tubo, según el requerimiento de prueba (carga constante y carga variable, subsistema 1 en Figura 1). En el otro extremo del flujómetro se colocó una manguera de alimentación de $1.27 \mathrm{~cm}$.

e) Fijación con prensa de presión. En una barra de $32 \mathrm{~cm}$ de solera de $1 \mathrm{~cm} x 1 \mathrm{~cm}$, se unieron en los extremos prensas de $5 \mathrm{~cm}, y$ también tres puntas perpendiculares a la barra de la misma solera, con el fin de sujetar los conductos dobles de alimentación con abrazaderas de $2.54 \mathrm{~cm}$. Las abrazaderas permiten subir o bajar todo el conjunto correspondiente a cada cilindro, lo que también ayuda a controlar las alturas de cargas de inundación y al mismo tiempo, variar, si se desea, la columna de agua dentro de cada cilindro, de forma independiente. 


\section{Ciencias ฐึgua}

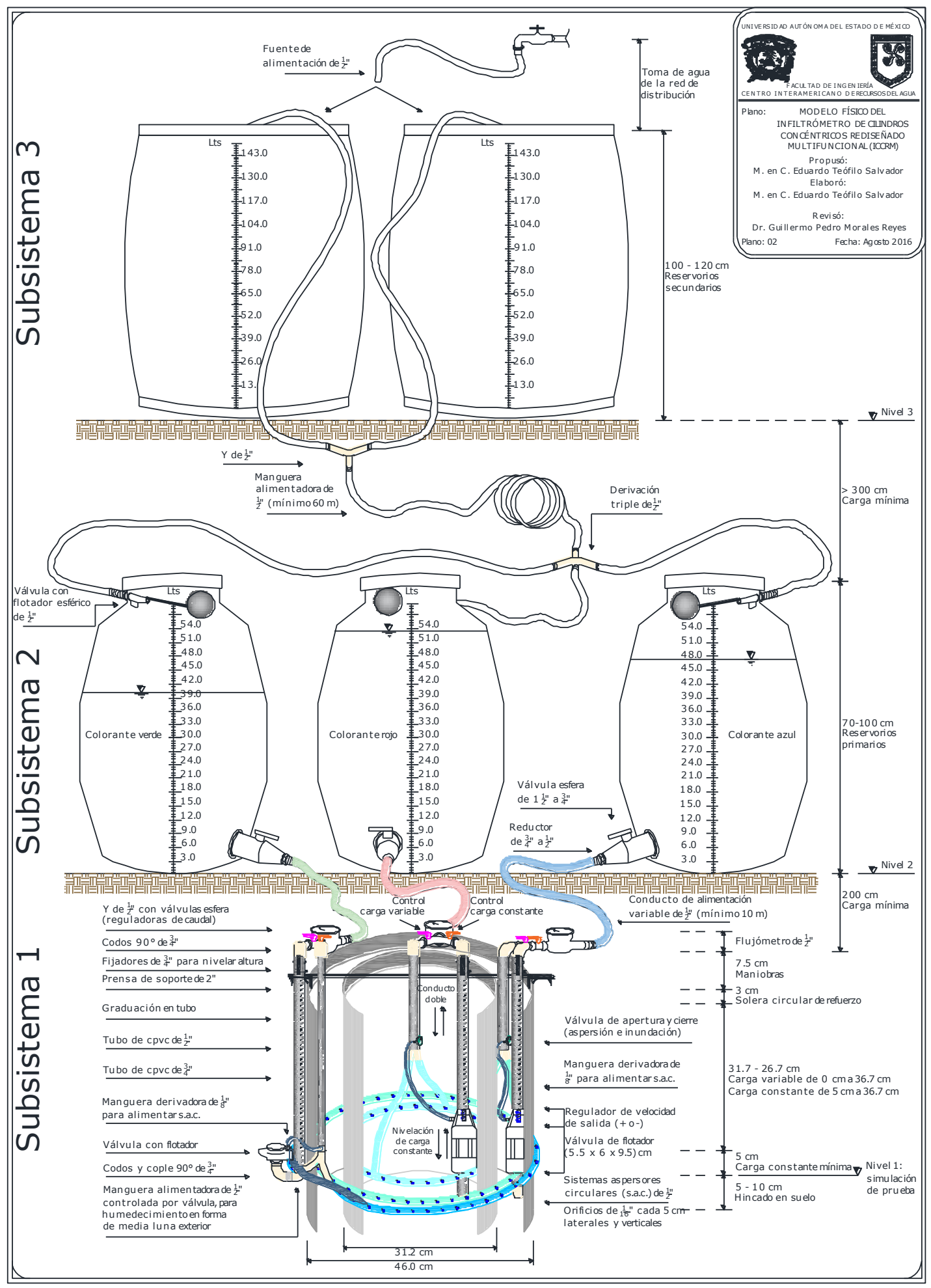


Figura 1. Modelo de infiltrómetro de cilindros concéntricos rediseñado multifuncional para funcionamiento con cambios de nivel (elaboración propia).

Subsistema 2. Reservorios primarios. Debido al requerimiento de agua, se consideró colocar tres reservorios primarios con capacidad de $60 \mathrm{~L}$, a una altura mayor de $2 \mathrm{~m}$, como se observó en la Figura 1; esto, para el buen funcionamiento del subsistema 1. En la parte inferior de cada reservorio se propuso una válvula esfera de $3.81 \mathrm{~cm}$ a $1.90 \mathrm{~cm}$, a partir de la cual se adapta una manguera de $1.27 \mathrm{~cm}$ para alimentar a cada flujómetro, con una longitud mínima de $10 \mathrm{~m}$. En la parte superior de cada recipiente se coloca una válvula de $1.27 \mathrm{~cm}$ con flotador para regular el llenado, y evitar cambiar constantemente la manguera de suministro, para ello se planteó un cople de derivación triple para tener controlados los tres reservorios de manera simultánea. Para identificar la distribución del flujo vertical, lateral y superficial por el movimiento del agua proveniente de cada reservorio se consideró utilizar trazadores: color verde para el aspersor exterior, azul para el intermedio y el rojo para el interior (subsistema 2 en Figura 1).

Subsistema 3. Reservorios secundarios. Son dos recipientes de $150 \mathrm{~L}$ cada uno y que se colocan a un nivel superior al de los reservorios primarios, con una carga mayor a $3 \mathrm{~m}$. De estos reservorios se deriva una manguera de $1.27 \mathrm{~cm}$, con un cople tipo $Y$ para retardar el tiempo de vaciado, después se coloca una manguera de $1.27 \mathrm{~cm}$, con una longitud considerable. Es importante resaltar que los reservorios secundarios deben estar llenándose continuamente de la toma domiciliaría, como se ilustra en la Figura 1, para no interrumpir el caudal de alimentación durante las pruebas de campo.

\section{Construcción}

Se cuantificó y adquirió el material necesario para la construcción, se presentaron las piezas como se observa en la Figura 2a. Posteriormente inició la revisión de la altura de las válvulas con flotador dentro de los cilindros, para lo cual se recortó un sobrante de estas válvulas en la 
parte inferior, y se ajustó con codos de $90^{\circ}$ para obtener la mínima altura de control de carga constante. Se adaptaron los flujómetros con la derivación tipo $Y$ de $1.27 \mathrm{~cm}$, y se unieron a un solo tubo de CPCV de $1.90 \mathrm{~cm}$, para revisar el funcionamiento de flujómetros y válvulas, tal y como se ilustra en la Figura $2 b$.
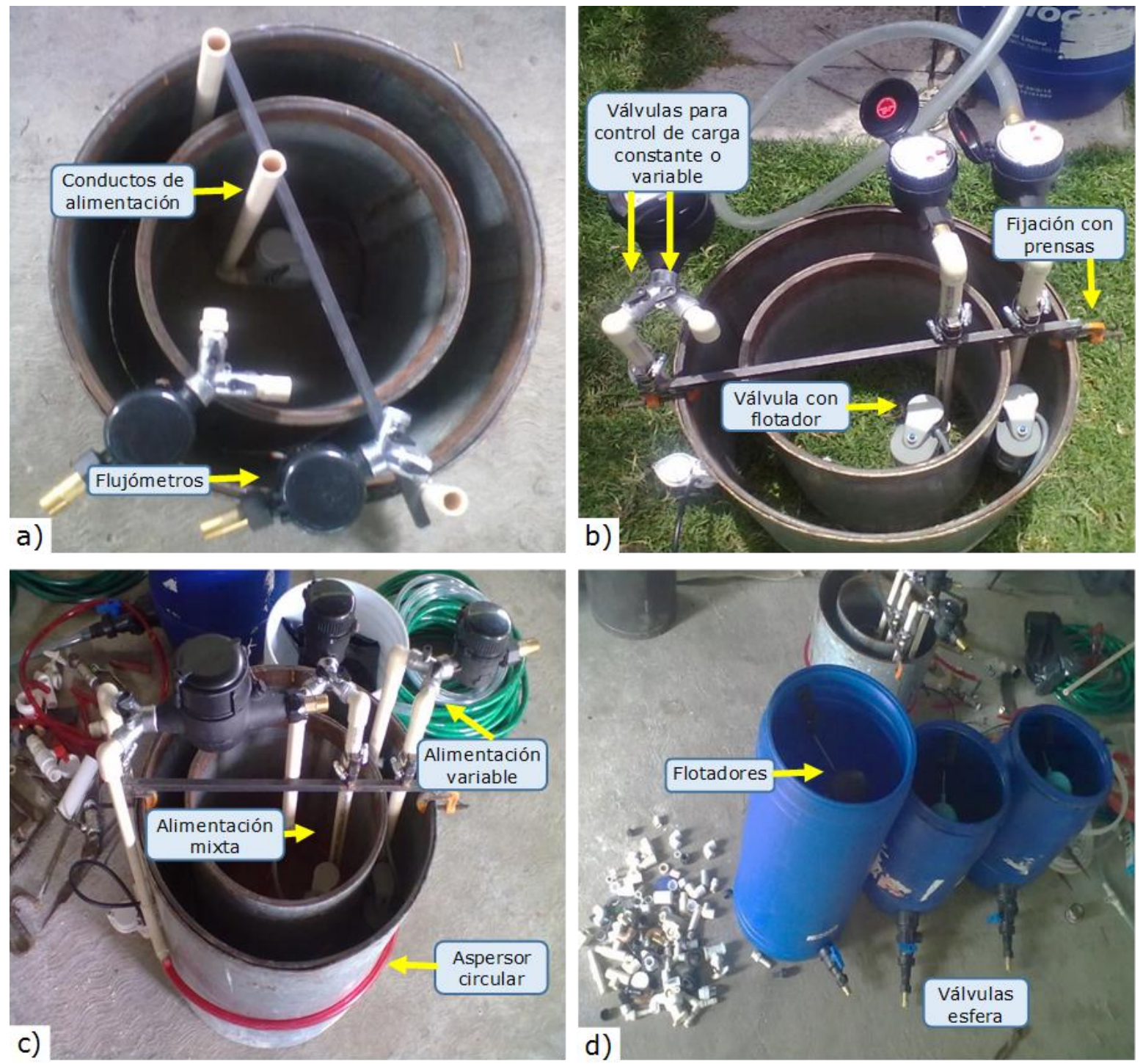

Figura 2. Procedimiento constructivo: a) adaptación de flujómetros y válvulas con flotador; b) revisión de fugas de agua; c) sistemas aspersores acoplados a los conductos de alimentación, y d) adaptaciones de los flotadores y válvulas esfera a los reservorios primarios. 
A continuación se construyeron los aspersores circulares de 25, 35 y $48 \mathrm{~cm}$ de diámetro con manguera de $1.27 \mathrm{~cm}$ de diámetro, y se adaptaron al otro conducto de alimentación de $1.90 \mathrm{~cm}$ con la miniválvula de paso (Figura $2 \mathrm{c}$ ), el cual no se pegó a la derivación tipo Y, para facilitar la instalación. Consecutivamente, se adaptaron las válvulas con flotador y válvulas esfera a los recipientes primarios (Figura 2d); asimismo, se construyó un cople con derivación triple para conectar 3 ramales de manguera de $1.27 \mathrm{~cm}$.

Es importante mencionar que se optó por ocupar un reservorio primario de $100 \mathrm{~L}$, y dos de $60 \mathrm{~L}$, el primero asignado a humedecimiento exterior, ya que el área de contacto es mayor que el área dentro de los cilindros. También se adaptaron coples de manguera para reducir de $1.90 \mathrm{~cm}$ a $1.27 \mathrm{~cm}$, y durante el procedimiento constructivo se revisaron fugas de agua con el fin de detectarlas a tiempo y repararlas antes de seguir ajustando y pegando piezas. Finalmente, para los reservorios secundarios se construyeron dos arnés de varilla de diámetro $0.95 \mathrm{~cm}$ por separado, para contener a cada uno de estos recipientes, debido al volumen contenido y para facilidad de transporte, así como para almacenar suficiente volumen de agua.

\section{Verificación funcional}

La verificación funcional del infiltrómetro de cilindros concéntricos rediseñado multifuncional, tuvo por objetivo revisar el funcionamiento de todo el sistema, desde el punto de prueba, hasta los reservorios secundarios; por ello la experimentación se realizó en un terreno con pendiente significativa. Primero se llenaron los reservorios secundarios y se instalaron los primarios (Figura 3 a y 3 b), secuencialmente se instalaron los conductos, se llenaron los reservorios primarios y se agregaron los trazadores de color en cada recipiente. A continuación, se hincaron los cilindros y se acoplaron los accesorios construidos (Figura 3c). Para iniciar la prueba se observó la lectura inicial en cada flujómetro y se abrieron las válvulas esfera de la bifurcación tipo Y. Se empezó con una reproducción constante de $50 \mathrm{~mm} / \mathrm{min} / \mathrm{m}^{2}$ para los cilindros y fuera de éstos, por lo que se realizó una proporción en función del área de contacto del agua con el suelo. Asimismo se comparó el funcionamiento del modelo propuesto con el uso del infiltrómetro de cilindros concéntricos tradicional (Figura 3e) con una carga hidráulica de 165 mm, en el mismo sitio de prueba. En adición se 
muestreo el suelo para determinar la clase textural, y con ello se aplicó el método de las curvas de infiltración establecidas por el Soil Conservation Service (SCS) para diferentes tipos de suelo (Subramanya, 2008), con la finalidad de comparar los resultados obtenidos.
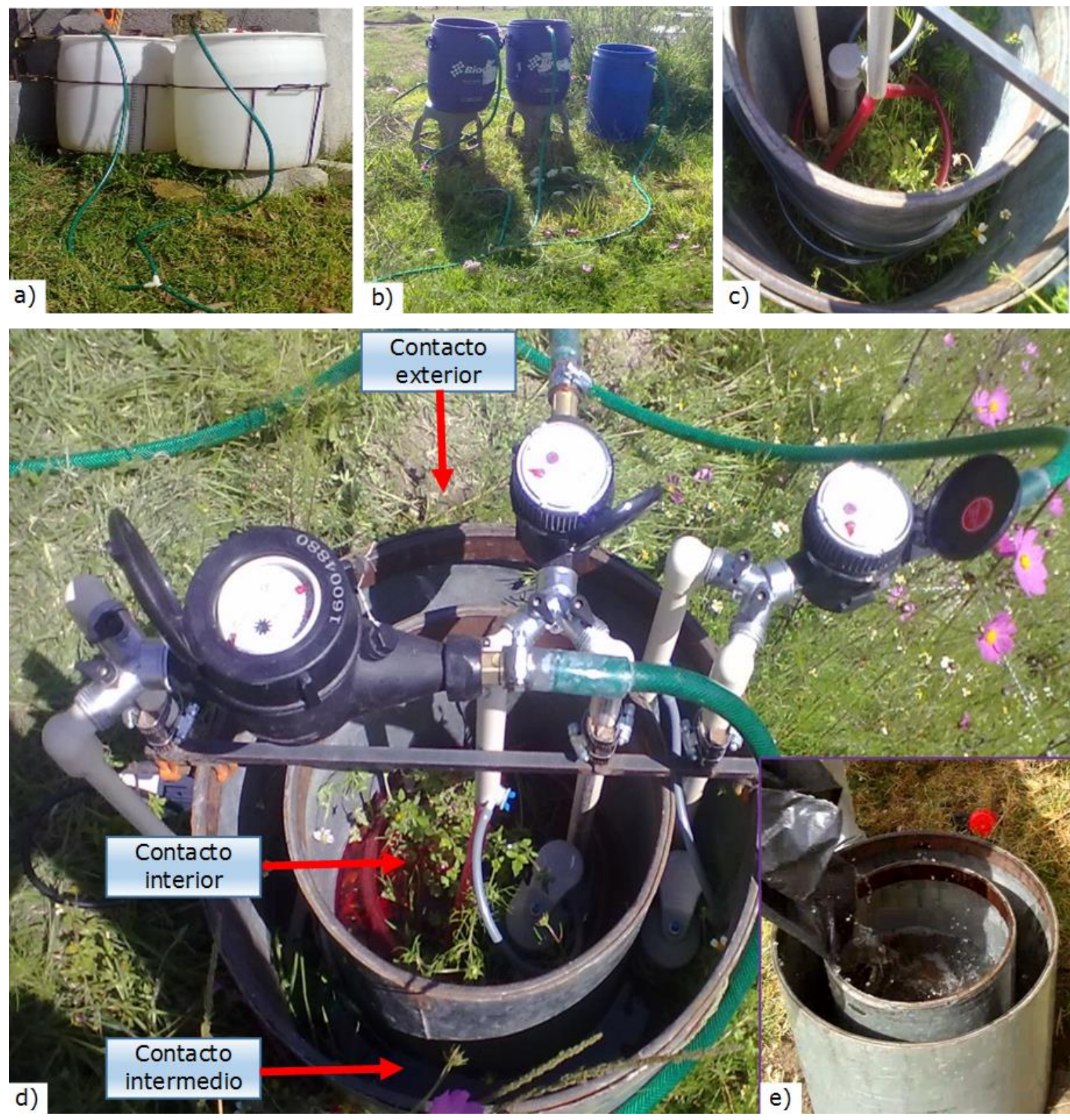

Figura 3. Verificación funcional del modelo propuesto: a) reservorios secundarios; b) reservorios primarios; c) sistemas aspersores; d) infiltrómetro de cilindros concéntricos rediseñado 
multifuncional; e) infiltrómetro de cilindros concéntricos tradicional.

\section{Ajuste de datos con el modelo de Kostiakov}

De la medición de descensos, para lecturas constantes se realizó una distribución del descenso conocido entre el lapso en el que se presentó, con el fin de evitar la presencia de valores nulos en las tablas de captura, esto es:

$$
h_{i+1}=h_{i}+\left(\frac{h_{n}-h_{i}}{t_{n}-t_{i}}\right)\left(t_{i+1}-t_{i}\right)
$$

Donde, $h_{i+1}$ es el nivel de agua desconocido para el tiempo $t_{i+1} ; h_{i}$ es el nivel de agua registrado en el tiempo $t_{i} ; h_{n}$ es el nivel de agua registrado en el tiempo $t_{n}$, así la distribución ocurre de $i \geq 1$ hasta $i=n$ 2 ; y $\mathrm{n}$ es el número de intervalos, por lo que la distribución se realizó para valores intermedios a partir de los valores extremos (inferior y superior) conocidos. Con los datos distribuidos se determinó la tasa de infiltración $\left(\Delta h_{i} / \Delta t_{i}\right)$, así como la infiltración acumulada $\left(\Delta H_{i}=\Delta h_{i}+\Delta h_{i-}\right.$ 1), para ambos cilindros (Teófilo, 2015). Posteriormente se ajustó la información al modelo de Kostiakov para representar el comportamiento de la tasa de infiltración e infiltración acumulada de la forma:

$$
f=\alpha t^{\beta}
$$

Para $t \neq 0, a>0$ y $-1<b<0$; donde $t$ es el tiempo; $a$ es el coeficiente; b es el exponente, y $f$ la tasa de infiltración. Al integrar la ecuación entre los límites $t=0$ y $t=t$, se obtiene la ecuación de la infiltración acumulada $(F)$, quedando de la siguiente forma:

$$
F=\frac{\alpha}{(\beta+1)} t^{\beta+1}
$$

\section{Resultados}




\section{Funcionamiento del modelo de infiltrómetro de cilindros concéntricos rediseñado multifuncional (ICCRM)}

Con respecto a la nueva propuesta de diseño, revisión y construcción del infiltrómetro de cilindro concéntricos rediseñado multifuncional se encontró que:

1. Para un funcionamiento adecuado, desde la ubicación del infiltrómetro multifuncional, hasta los reservorios primarios o secundarios, el vertido por gravedad para garantizar las cargas hidráulicas mínimas de operación de $2 \mathrm{~m}$ debe ser considerado.

2. Para simular algún evento es necesario definir previamente las condiciones, es decir inundación puntual, por aspersión, de carga constante, de carga variable o combinada, en cualquiera de las tres áreas de contacto con el suelo.

3. La instalación y operación de todo el sistema requiere de control y conocimiento total del equipo, de los subsistemas, de la recolección de lecturas y de la identificación del comportamiento de las trayectorias del flujo en forma simultánea.

En la Tabla 1 se muestran las formas en que se puede utilizar el nuevo modelo físico de infiltrómetro multifuncional, ya que cuenta tanto de conexión de caída directa de agua de las válvulas de flotador, como de los aspersores circulares, que están conectados al conjunto de doble de alimentación, por ello es posible utilizarlo de ambas formas o bien combinado. De esta forma, debido a los flujómetros, es posible controlar el gasto de salida a flujo constante o variable, o un combinado de éstos.

Tabla 1. Usos del infiltrómetro de cilindros concéntricos rediseñado multifuncional (elaboración propia).

\begin{tabular}{|c|c|c|c|c|c|c|}
\hline Uso/control & $\begin{array}{c}\text { Inundación } \\
\text { puntual }\end{array}$ & $\begin{array}{c}\text { Inundación } \\
\text { por } \\
\text { aspersión }\end{array}$ & $\begin{array}{c}\text { Altura } \\
\text { operable }\end{array}$ & $\begin{array}{l}\text { Contacto } \\
\text { interior }\end{array}$ & $\begin{array}{c}\text { Contacto } \\
\text { intermedio }\end{array}$ & $\begin{array}{l}\text { Contacto } \\
\text { exterior }\end{array}$ \\
\hline $\begin{array}{l}\text { Carga } \\
\text { constante }\end{array}$ & si & si & 5 a $30 \mathrm{~cm}$ & si & si & \\
\hline $\begin{array}{l}\text { Carga } \\
\text { variable }\end{array}$ & si & si & 0 a $30 \mathrm{~cm}$ & si & si & \\
\hline $\begin{array}{l}\text { Carga } \\
\text { descendente }\end{array}$ & si & si & 30 a $5 \mathrm{~cm}$ & si & si & \\
\hline Carga & si & si & 5 a $30 \mathrm{~cm}$ & si & si & \\
\hline
\end{tabular}

Tecnología y ciencias del agua, 9(5), 103-131, DOI:10.24850/j-tyca-2018-06-05 


\begin{tabular}{|c|c|c|c|c|c|c|}
\hline $\begin{array}{l}\text { Carga } \\
\text { combinada }\end{array}$ & si & si & 0 a $30 \mathrm{~cm}$ & si & si & \\
\hline $\begin{array}{l}\text { Gasto } \\
\text { constante }\end{array}$ & si & si & & & & si \\
\hline $\begin{array}{l}\text { Gasto } \\
\text { variable }\end{array}$ & si & si & & si & si & si \\
\hline $\begin{array}{l}\text { Gasto } \\
\text { combinado }\end{array}$ & si & si & & si & si & si \\
\hline
\end{tabular}

En la Tabla 1 se observa que para el área de contacto exterior no es posible mantener una carga de inundación constante, debido a que la superficie exterior expuesta no está limitada, por lo que la carga o flujo puede generar escurrimiento, o un bulbo local de humedecimiento; sin embargo, con ello es posible humedecer la capa evaporante que con la metodología tradicional no se lograba. Además, de que con este nuevo modelo es posible medir el comportamiento de la infiltración vertical, lateral y superficial para un mismo tipo de suelo.

\section{Comportamiento de los datos ajustados}

La buena de operación del nuevo modelo de infiltrómetro permitió recabar el mayor número de lecturas en los intervalos de tiempo de la prueba, de esta forma se recolectaron tres comportamientos de campo; infiltración vertical, lateral y superficial. El comportamiento de cada infiltración se muestra en la Figura 4, para un material de textura arenosa hasta una profundidad de $0.60 \mathrm{~m}$, donde los primeros $0.05 \mathrm{~m}$ a partir de la superficie se encontraron secos con presencia de pastizal. 


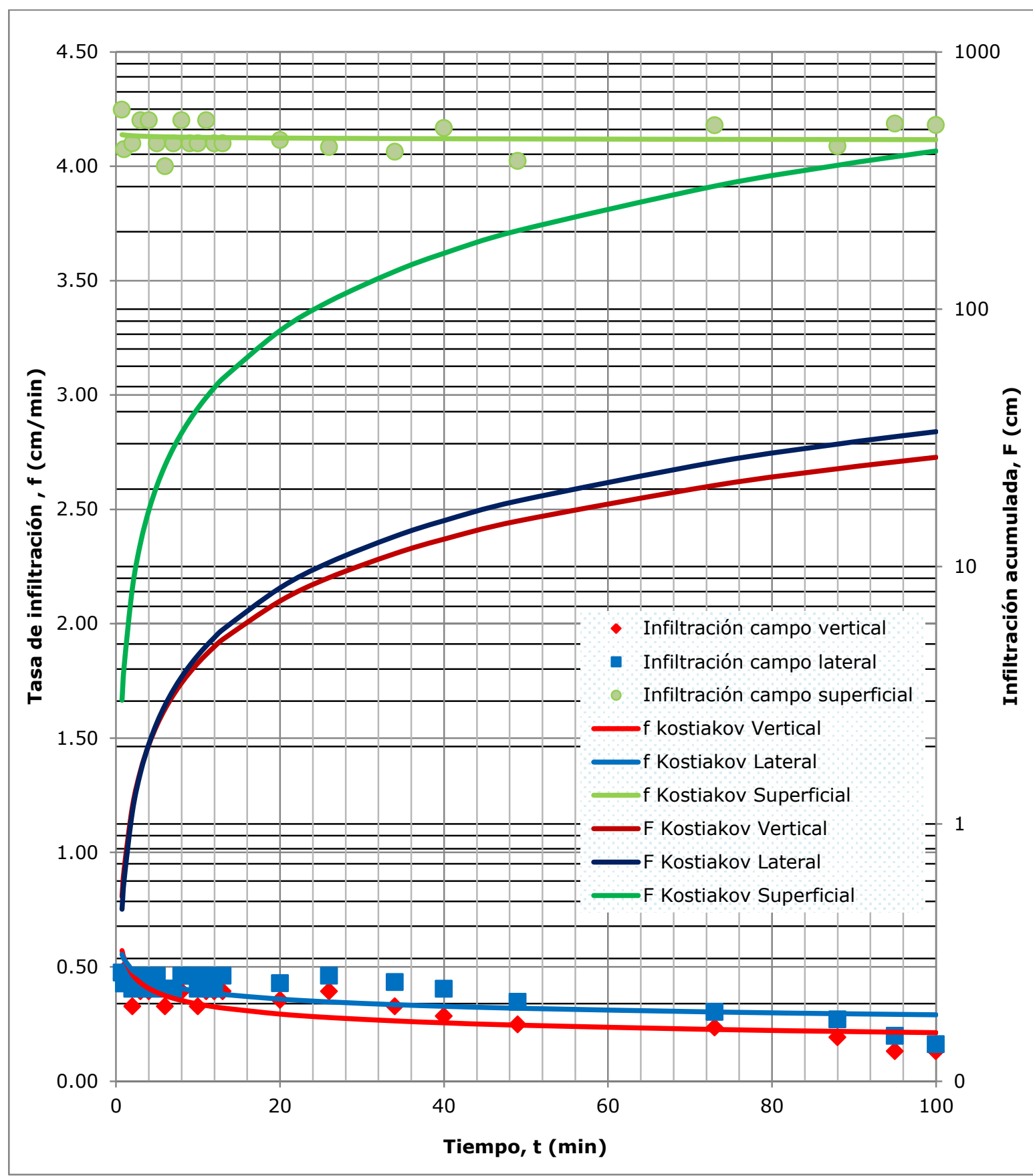

Figura 4. Comportamiento de la infiltración vertical, lateral y
superficial para una simulación constante de $50 \mathrm{~mm} / \mathrm{min} / \mathrm{m}^{2}$.

En la Figura 4 se muestra la tasa de infiltración exterior constante durante el periodo de prueba, además de la tasa de infiltración vertical y Tecnología y ciencias del agua, 9(5), 103-131, DOI:10.24850/j-tyca-2018-06-05 
lateral para la simulación de $50 \mathrm{~mm} / \mathrm{min} / \mathrm{m}^{2}$, siendo estas dos últimas complementarias a la tasa superficial. Sin embargo, se puede apreciar que como el agua vertida en los cilindros es constante, al inicio permite determinar una tasa máxima de $5 \mathrm{~mm} / \mathrm{min}$, pero a continuación, la limitación de los cilindros genera que el comportamiento de la tasa de infiltración se reduzca aproximadamente a $2 \mathrm{~mm} / \mathrm{min}$, debido al efecto del bulbo de humedecimiento fuera de los cilindros.

Cabe resaltar que de esta forma fue posible generar el proceso de saturación y sobresaturación de la masa de suelo, además con este nuevo modelo de infiltrómetro es posible aproximar un valor del escurrimiento, es decir en el caso exterior la infiltración acumulada fue de $412 \mathrm{~cm}$, de $26 \mathrm{~cm}$ en vertical y de 34 en lateral, cuya suma de estas dos últimas fue de $60 \mathrm{~cm}$, que representa el $14.56 \%$ del obtenido en la superficie fuera de los cilindros, por lo que con esas condiciones y pensando que el agua exterior se mueve vertical, lateral y superficialmente, el escurrimiento aproximado fue de $85.44 \%$, siendo un valor significativo debido a la intensidad simulada.

\section{Discusión}

Con respecto a la aplicación del método de las curvas de infiltración SCS-USDA se consideró el grupo de suelo $A$, cobertura pastizal, sin tratamiento agrícola y pendiente de $30^{\circ}$ de inclinación, como condiciones físicas observables y medibles en campo, con lo que se estimó un escurrimiento del $72.02 \%$. La diferencia con respecto al $85.44 \%$, obtenido antes, se atribuye a la técnica aplicada y a la escala de aplicación. De esta forma se comprueba que el modelo propuesto es aceptable, si se cuenta con un conocimiento adecuado del sistema y operación. Al mismo tiempo, varios autores recomiendan pruebas de campo, pues son más realistas para reproducir un fenómeno, sobre todo por los cambios dinámicos (Sassa, Fukuoka, Ochiai, Wang \& Wang 2005; Velázquez, Mas, Mayorga, Díaz, Alcántara, Castro, Fernández, Palacio, Bocco, Gómez, Luna, Trejo, López, Palma, Peralta, Prado \& González, 2002).

Tecnología y ciencias del agua, 9(5), 103-131, DOI:10.24850/j-tyca-2018-06-05 
Con respecto a la comparación del funcionamiento del infiltrómetro de cilindros concéntricos tradicional con el infiltrómetro de cilindros concéntricos rediseñado multifuncional. En la Figura 5 se muestra el comportamiento de las lecturas de campo, la tasa de infiltración y la infiltración acumulada, ajustados con el modelo de Kostiakov, esto relacionado con la distribución del agua por aspersión en el suelo. Los valores iniciales de campo de ajuste de tasa de infiltración e infiltración acumulada del ICC rediseñado multifuncional son mayores, y éstos son los más representativos en todo el periodo de prueba, también las fluctuaciones se reducen en la compensación de descensos, debido al reacomodo del agua en el suelo, ya que se vierte de manera gradual. 

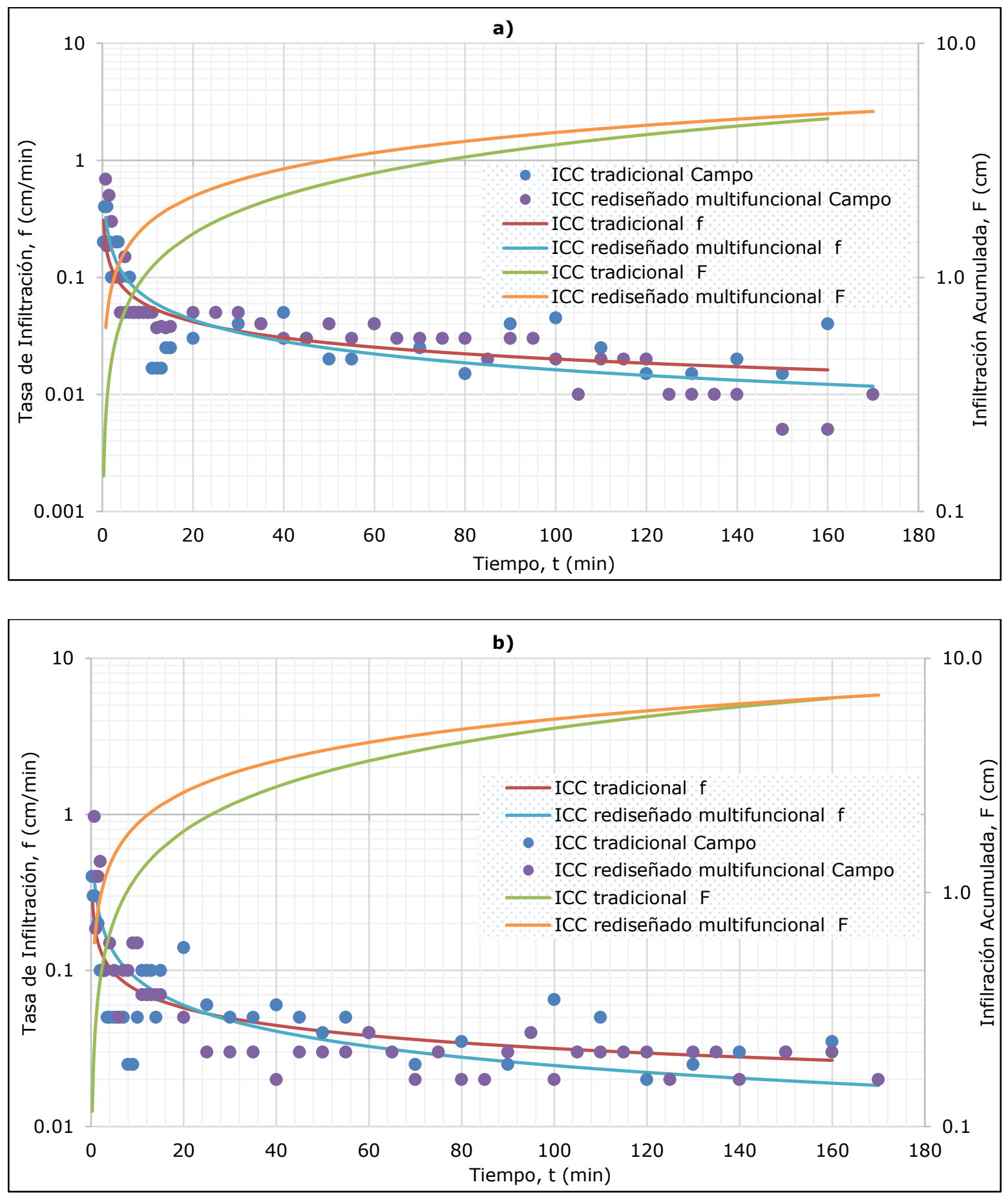

Figura 5. Comparación del comportamiento de datos experimentales del infiltrómetro de cilindros concéntricos tradicional (ICC) y con el infiltrómetro de cilindros concéntricos rediseñado multifuncional (ICCRM) para una distribución de agua por aspersión; a) cilindro interior; b) cilindro exterior.

Tecnología y ciencias del agua, 9(5), 103-131, DOI:10.24850/j-tyca-2018-06-05 
Además, en la Figura 5, se observa que el comportamiento de la tasa de infiltración después del minuto 20 es muy parecida para ambos equipos. De la misma manera, del minuto 0 al 20, la velocidad de infiltración del equipo rediseñado multifuncional es mayor, tanto para el cilindro interior como para el exterior. La diferencia entre las dos curvas de infiltración acumulada, se debe a la heterogeneidad del suelo, así como al tiempo de respuesta para saturar al mismo, pero esencialmente, al cambio de presión (por inundación gradual y de impacto), siendo esta diferencia muy importante para aproximar adecuadamente procesos como es la infiltración.

En la Tabla 2 se presentan los parámetros más sobresalientes del comportamiento de los registros de ambos equipos. El margen de error para alcanzar una lámina deseada $\left(h_{\mathrm{wc}}\right)$ de $165 \mathrm{~mm}$, fue del 0 al $1 \%$ para el rediseñado multifuncional y del 0 al $6 \%$ para el tradicional. La tasa inicial $\left(f_{1}\right)$ del rediseñado va de 140 a $240 \%$ mayor al tradicional, ya que se conoce la cantidad de agua que se infiltra de $t_{0}$ a $t_{1}$. Con respecto al coeficiente de correlación, el mejor ajuste se tiene con el rediseñado multifuncional, cuya diferencia es del 16 a 33\%, en comparación con el tradicional, pues representa mejor en representar el proceso de la infiltración.

\section{Tabla 2. Parámetros comparativos del ICC tradicional y el ICC rediseñado multifuncional.}

\begin{tabular}{|c|c|c|c|c|c|c|}
\hline \multirow[b]{2}{*}{ Medición } & \multicolumn{5}{|c|}{ Infiltrómetro de cilindros concéntricos } & \multirow[b]{2}{*}{ Unidad } \\
\hline & \multicolumn{2}{|c|}{ ICC Tradicional } & \multicolumn{3}{|c|}{ ICC Rediseñado multifuncional } & \\
\hline Cilindro & Interior & Exterior & Interior & Intermedio & Exterior & \\
\hline$h_{w c}$ & 17.50 & 16.00 & 16.50 & 16.65 & -- & $\mathrm{cm}$ \\
\hline$\Delta \mathrm{h}$ (infiltrada inicial) & -- & -- & 0.50 & 0.70 & -- & $\mathrm{cm}$ \\
\hline Tiempo de Ilenado & \multicolumn{2}{|c|}{2.5 (ambos) } & 0.73 & 0.73 & -- & $\min$ \\
\hline$f_{1}$ (inicial) & 0.2 & 0.4 & 0.68 & 0.96 & & $\mathrm{~cm} / \mathrm{min}$ \\
\hline$f_{1}$ (ajuste) & $0.1639 t^{-0.456}$ & $0.1746 t^{-0.37}$ & $0.2691 t^{-0 .}$ & $0.3135 t^{-0.553}$ & & $\mathrm{~cm} / \mathrm{min}$ \\
\hline$F_{1}$ (ajuste) & $0.3013 t^{0.543}$ & $0.2776 t^{0.62}$ & $0.6897 t^{0.3}$ & $0.7012 t^{0.447}$ & & $\mathrm{~cm}$ \\
\hline$r^{2}$ & 0.69 & 0.62 & 0.80 & 0.82 & & \\
\hline
\end{tabular}

La desventaja en utilizar el equipo tradicional es que no se conoce el volumen de agua que infiltra antes de comenzar las mediciones,

Tecnología y ciencias del agua, 9(5), 103-131, DOI:10.24850/j-tyca-2018-06-05 
siendo este volumen, el que determina el comportamiento inicial de las curvas de infiltración e infiltración acumulada. Para este caso, no considerar este volumen equivale a ignorar precipitaciones de 5 a $7 \mathrm{~mm}$, las cuales son esenciales para humedecimiento inicial del suelo, inundaciones eventuales y control de cultivos.

En un medio poroso, la lámina de agua dentro de los cilindros puede variar en función de la humedad, textura y compactación del suelo. Para suelos como las arcillas o muy compactos, la lámina de infiltración de $t_{0}$ a $t_{1}$ será relativamente pequeña, en comparación con los suelos arenosos (Teófilo, 2010). Por lo tanto, y para fines prácticos, la lámina máxima de las descargas de los reservorios es adecuada en el uso del ICCRM, pues se controla de manera simultánea para ambos cilindros.

En primera instancia, se ha comparado experimentalmente, en pruebas de campo el nuevo modelo, con base en la metodología tradicional, donde existe cierta incertidumbre debido a variabilidad espacial y temporal de las condiciones del suelo, así como a la estructura y operación del nuevo equipo, lo que le da mayor ventaja sobre el tradicional. De esta forma, para posteriores trabajos, se debe tomar en cuenta una calibración numérica con HYDRUS, ya que este modelo ha sido adecuado en la simulación de la infiltración con el infiltrómetro de cilindros concéntricos (Lai et al., 2010; Lai y Ren, 2007).

\section{Conclusiones}

El infiltrómetro multifuncional surge de la necesidad de mejorar la reproducción de procesos naturales como la precipitación y de ésta el escurrimiento, así como provocar el flujo vertical, lateral y superficial en el suelo. Además, este equipo permite controlar una carga hidráulica constante, variable, ascendente, descendente, mixta o combinada, así como un gasto constante, variable o combinado. Con el nuevo infiltrómetro multifuncional se pueden reproducir precipitaciones en función de la intensidad, duración, frecuencia y distribución, con igualdad de aplicación para los tres sectores de contacto con el suelo.

Tecnología y ciencias del agua, 9(5), 103-131, DOI:10.24850/j-tyca-2018-06-05 
A partir de lo anterior, se pueden determinar las componentes; tasa de infiltración vertical, lateral y el humedecimiento de la capa evaporante, que en la metodología tradicional no se realizaba, por lo que la medición de este parámetro puede ser relacionado con el comportamiento exterior, con lo cual es posible aproximar el escurrimiento superficial.

Con los ajustes realizados en la metodología tradicional de los cilindros concéntricos para su mejora, y en función de los resultados, se ha obtenido que se disminuye la alteración a la superficie del suelo por el agregado de agua de manera uniforme, sobre toda la superficie de contacto. También se reduce el tiempo de vertido para alcanzar una columna de agua dentro de los cilindros de 3 minutos a 53 segundos, siendo $70 \%$ más rápido que el infiltrómetro tradicional. También, se alcanza la misma lámina de agua para ambos cilindros en un tiempo $t_{1}$, por lo que se evitan flujos de compensación, se reducen las ondulaciones del agua que se generan con el uso del equipo tradicional al retirar los hules, y se puede determinar la cantidad de agua inicial que infiltra (de $t_{0}$ a $t_{1}$ ), en el tiempo que se alcanza la lámina requerida dentro de los cilindros.

Con respecto al tratamiento de los registros de la práctica, los valores iniciales en la curva de la tasa de infiltración son mayores de 140 a $240 \%$, en comparación con la tradicional, debido a que ya se conoce la cantidad de agua infiltrada en el tiempo de vertido, por lo que subestimar la lámina de agua infiltrada al inicio, equivale a no considerar una precipitación de 5 a $7 \mathrm{~mm}$ sobre la superficie del suelo, y que progresivamente pudiera afectar el comportamiento del agua en el suelo. De igual forma, la diferencia entre las curvas de infiltración acumulada para ambos equipos se debe al reacomodo del agua en el suelo, debido a un cambio gradual de presión. Además, cuando la lámina de agua dentro de ambos cilindros es muy parecida y no hay cambios de presión por compensación, existe un mejor ajuste de los datos de campo, con el modelo de Kostiakov en el ICCRM, de 16 a 33\%, respecto al tradicional.

Se recomienda aplicar el equipo en otras áreas de estudio, considerando cambios de cobertura vegetal, topografía, textura de suelo, reproducción de precipitaciones o riego por inundación para ampliar la aplicabilidad en otras disciplinas y con ello comparar con otros equipos. Además de tener en cuenta que se requiere operar el equipo con una carga mínima de $2 \mathrm{~m}$ para obtener resultados confiables. 


\section{Agradecimientos}

Al Consejo Nacional de Ciencia y Tecnología, y al Centro Interamericano de Recursos del Agua de la Universidad Autónoma del Estado de México, por el apoyo brindado durante la realización del trabajo de investigación.

\section{Tablas}

Tabla 1. Usos del infiltrómetro de cilindros concéntricos rediseñado multifuncional (elaboración propia).

Tabla 2. Parámetros comparativos del ICC tradicional y el ICC rediseñado multifuncional.

\section{Imágenes o figuras}

Figura 1. Modelo de infiltrómetro de cilindros concéntricos rediseñado multifuncional para funcionamiento con cambios de nivel (elaboración propia).

Figura 2. Procedimiento constructivo: a) adaptación de flujómetros y válvulas con flotador, b) revisión de fugas de agua,

c) sistemas aspersores acoplados a los conductos de alimentación y d) adaptaciones de los flotadores y válvulas esfera a los reservorios primarios.

Figura 3. Verificación funcional del modelo propuesto: a) reservorios secundarios, b) reservorios primarios, c) sistemas aspersores, d) infiltrómetro de cilindros concéntricos rediseñado

multifuncional, e) infiltrómetro de cilindros concéntricos tradicional.

Figura 4. Comportamiento de la infiltración vertical, lateral y superficial para una simulación constante de $50 \mathrm{~mm} / \mathrm{min} / \mathrm{m}^{2}$.

Figura 5. Comparación del comportamiento de datos experimentales del infiltrómetro de cilindros concéntricos tradicional (ICC) y con el infiltrómetro de cilindros concéntricos rediseñado multifuncional (ICCRM) para una distribución de agua por aspersión; a) cilindro interior, b) cilindro exterior.

$$
\begin{array}{r}
\text { Ecuaciones } \\
h_{i+1}=h_{i}+\left(\frac{h_{n}-h_{i}}{t_{n}-t_{i}}\right)\left(t_{i+1}-t_{i}\right)
\end{array}
$$




$$
\begin{gathered}
f=\alpha t^{\beta} \\
F=\frac{\alpha}{(\beta+1)} t^{\beta+1}
\end{gathered}
$$

\section{Referencias}

Ahmed, M. M., Osman, M. M., \& Ibrahim, E. O. (2012). Note on double-ring infiltrometer with a water tank fitted with a float and a side manometer. Sudan Journal Dessertification Research, 4(1), 143-148.

Ahuja, L. R., El-Swaify, S. A. \& Rahman, A. (1976). Measuring hydrologic properties of soil with a double-ring infiltrometer and multiple-depth tensiometers. Soil Science Society of American Journal, 40, 494-499.

Álvarez, A. M., Carral, P., Hernández, Z., \& Almendros, G. (2013). Assessment of soil organic matter molecular characteristics related to hydrophysical properties in semiarid soils (Central Spain). Arid Land Research and Management, 27(4), 303-326. DOI: $10.1080 / 15324982.2013 .784376$

Andrade, R., \& Muralidharan, D. (2011). The influence of litho-stratification on the infiltrating water front in a granite terrain. Hidrological Sciences Journal, 56(5), 907-915.

Arriaga, F. J., Kornecki, T. S., Balkcom, K. S. \& Raper, R. L. (2010). A method for automating data collection from a double-ring infiltrometer under falling head conditions. Soil Use and Management, 26, 61-67. DOI: $10.1111 / j .1475-2743.2009 .00249 . x$.

ASTM, American Society for Testing and Materials. (1994). Standard test method for infiltration rate of soil in field using double-ring infiltrometer (informe D 3385-9403), United States

Bouwer, H. (1986). Intake rate: cylinder infiltrometer. En: klute, A. (eds.), Methods of soil Analysis (pp. 825-844). United Sates of America: Soil Science Society of America, American Society of Agronomy Part $1,2^{\text {nd }}$ ed. Agron. Monogr. 825-844. https://dl.sciencesocieties.org/publications/books/tocs/sssabookseries/ methodsofsoilan1

Chen, S. K., \& Wuing, L. C. (2002). Analysis of water movement in paddy rice fields (I) experimental studies. Journal of Hydrology, 260(1), 206-215. DOI: S0022-1694(01)00615-1. 
Chin, D. A. (2008). Phenomenological models of hydrologic processes in south Florida. Journal of Hydrology, 349(1-2), 230-243. DOI: 10.1016./j.jhydrolo.2007.11.003.

Chowdary, V. M., Damodhara, R. M., \& Jaiswal, C. S. (2006). Study of infiltration process under different experimental conditions. Agricultural Water Management, 83(1-2), 69-78. DOI:10.1016/j.agwat.2005.09.001.

Constantz, J. \& Murphy, F. (1987). An automated technique for flow measurements from Mariotte reservoirs. Soil Science Society of American Journal, 51, 252-254.

El-Hames, A. S., \& Al-Wagdany, A. S. (2013). Investigation of wetting front behavior due to rainfall and ponding head effects in arid región wadi alluvion. Arabian Journal of Geosciences, 6(5), 1499-1507. DOI: $10.1007 / \mathrm{s} 12517-011-0457-6$.

Fatehnia, M. \& Tawfiq, K. (junio 2014). Deriving vertical saturated hydraulic conductivity of soil using double ring infiltrometer infiltration information. $7^{\text {th }}$ International Conference on Environmental Science and Technology. Houston, Texas, vol. 2, pp. 224-230.

Fatehnia, M., Paran, S., Kish, S., \& Tawfiq, K. (2016). Automating double ring infiltrometer with an arduino microcontroller. Geoderma, 262, 133-139. Recuperado de https://doi.org/10.1016/j.geoderma.2015.08.022

Fodor, N., Sándor, R., Orfanus, T., Lichner, L., \& Rajkai, K. (2011). Evaluation method dependency of measured saturated hydraulic conductivity. Geoderma, 165(1), 60-68. DOI:10.1016/j.geoderma.2011.07.004.

Gómez-Tagle, C. A., Gutiérrez, G. J. A., \& Zepeda, C. H. (2010). Dispositivo de automatización para un infiltrómetro de campo con funcionamiento de Mariotte. Terra Latinoamericana, 28(3), 193-202. Recuperado de http://www.redalyc.org/articulo.oa?id $=57316064001$.

Gregory, J. H., Dukes, M. D., Miller, G. L., \& Jones, P. H. (2005). Analysis of double-ring infiltration techniques and development of a simple automatic water delivery system. Applied Turfgrass Science. Recuperado de https://abe.ufl.edu/faculty/mdukes/pdf/stormwater/ATS-double-ringpaper.pdf.

Jialilang, T., Bo, Z., Tao, W., Xunquiang, C., Moerong, G., \& Henry, L. (2012). Subsurface flow processes in sloping cropland of purple soil. Journal of Mountain Science, 9(1), 1-9. DOI: 10.1007/s11629-012-2199-7.

Köhne, J. M., Alvez, J. J., Köhne, S., Tiemeyer, B., Lennartz, B., \& Kruce J. (2011). Double-ring and tension infiltrometer measurements of 
Tecnología y

Ciencias ฐ Agua

hydraulic conductivity and mobile soil regions. Pesquisa Agropecuaria Tropical, Goiania, 41(3), 336-347.

Lai, J. \& Ren, L. (2007). Assessing the size dependency of measured hydraulic conductivity using double-ring infiltrometer and numerical simulation. Soil Science Society of America Journal, 71(6), 1667-1675. DOI: 10.2136/sssaj2006.0227.

Lai, J., Luo, Y. \& Ren, L. (2010). Buffer index effects on hydraulic conductivity measurements using numerical simulations of double-ring infiltration. Soil Science Society of American Journal, 74(5), 1526-1536. DOI: 10.2136/sssaj2009.0450.

Lazarovitch, N., Ben-Gal, A., Šimůnek, J. \& Shani, U. (2007). Uniqueness of soil hydraulic parameters determined by a combined wooding inverse approach. Soil Science Society of American Journal, 71(3), 860-865. Recuperado de http://dx.doi.org/10.2136/sssaj2005.0420.

Lichner, L., Eldridge, D. J., Schacht, K., Zhukova, N., Holko, L., Sir, M., \& Pecho, J. (2011). Grass cover influences hydrophysical parameters and heterogeneity of water flow in a sandy soil. Pedosphere, 21(6), 719729.

Matula, S. \& Dirksen, C. (1989). Automated regulating and recording system for cylinder infiltrometer. Soil Science Society of America Journal, 53(1), 299-302. DOI:10.2136/sssaj1989.03615995005300010056x.

Milla, K. \& Kish, S. (2006). A low-cost microprocessor and infrared sensor system for automating water infiltration measurements. Computers and Electronics in Agriculture, 53, 122-129. DOI: $10.1016 /$ j.compag.2006.05.001.

Návar, J., \& Synnott, T. J. (2000). Soil infiltration and land use in Linares, N. L., Mexico. Terra Latinoamericana, 18(3), 255-262. Recuperado de http://www. redalyc.org/articulo.oa?id=57318309.

Neris, J., Jiménez C., Fuentes, J., Morillas, G. \& Tejedor, M. (2012). Vegetation and land-use effects on soil properties and water infiltration of andisols in Tenerife (Canary Islands, Spain). Catena, 98(6), 55-62. DOI: $10.1016 / j$.catena.2012.06.006.

Ong, J., Werkema, D. \& Lane, J. J. W. (2012). Revisiting the fully automated double-ring infiltrometer using open-source electronics. Recuperado de https://cfpub.epa.gov/si/si_public_record_report.cfm?dirEntryId=24825 1.

Pinheiro, A., Poeta, T. L., \& Kaufmann, V. (2009). Capacidade de infiltracao de agua em solos sob diferentes usos é prácticas de manejo agrícola. 
Tecnología y

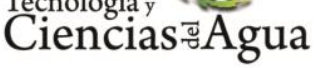

Ambiente y Agua - An Interdisciplinary Journal of Applied Science, 4(2), 188-199. Recuperado http://www.redalyc.org/articulo.oa?id=92811747017.

de

Raoof, M., Hosein, N. A., Ashraf, S. A., \& Marofi, S. (2011). Measuring and estimating saturated and unsaturated hydraulic conductivity in steady and transient states on sloping lands. World Applied Sciences Journal, 13(4), 747-755. Recuperado de http://www.idosi.org/wasj/wasj13(4)/19.pdf.

Reynolds, B., \& Reddy, K. J. (2012). Infiltration rates in reclaimed surface coal mines. Water, Air, \& Soil Pollut, 223(9), 5941-5958. DOI: $10.1007 / \mathrm{s} 11270-12-1330-2$.

Ronayne, M., Houghton, T., \& Stednick, J. (2012). Fiel characterization of hydraulic conductivity in a heterogeneous alpine glacial till. Journal of Hydrology, 458-459, 103-109.

Salas, G. J. (2012). Determinación espacial de la recarga mediante el diseño e instalación de instrumentación en pozos de monitoreo y simulación de la infiltración en la zona vadosa (tesis de doctorado). Universidad Autónoma del Estado de México, Toluca, México.

Sassa, K., Fukuoka, H., Ochiai, H., Wang, F. \& Wang, G. (2005). Aerial prediction of earthquake and rain induced rapid and long-traveling flow phenomena (APERITIF) (M101). En: Sassa K., Fukuoka, H., Wang F. \& G. Gonghui G. (eds.). Landslides Risk Analysis and Sustainable Disaster Management (pp. 99-108). Berlin, Alemania: Springer.

Sharratt, B., Zhang, M. \& Sparrow, S. (2006). Twenty years of conservation tillage research in subarctic Alaska II. Impact on soil hydraulic properties. Soil \& Tillage Research, 91(1-2), 82-88, DOI: $10.1016 /$ j.still.2006.01.010.

Subramanya, K. (2008). Engineering hydrology ( $3^{\text {er }}$ ed.). Nueva Delhi: McGraw-Hill.

Teófilo, S. E. (2010). Aplicación del infiltrómetro de cilindros concéntricos en la determinación aproximada de la velocidad de infiltración lateral (tesis de licenciatura). Universidad Autónoma del Estado de México, Toluca, México.

Teófilo, S. E. (2015). Propuesta metodológica para la estimación de la infiltración eficaz con uso del infiltrómetro de cilindros concéntricos rediseñado (tesis de maestría). Universidad Autónoma del Estado de México, Toluca, México.

Tecnología y ciencias del agua, 9(5), 103-131, DOI:10.24850/j-tyca-2018-06-05 
Touma, J., \& Albergel, J. (1992). Determining soil hydrologic properties from rain simulator or double ring infiltrometer experiments: a comparison. Journal of hydrology, 135, 73-86. Recuperado de http://horizon.documentation.ird.fr/exldoc/pleins_textes/pleins_textes_6/b_fdi_43-44/010004945.pdf.

Velázquez, A., Mas, J. F., Mayorga, S. R., Díaz, J. R., Alcántara, C., Castro, R., Fernández, T., Palacio, J. L., Bocco, G., Gómez, R. G., Luna, G. L., Trejo, I., López, G. J., Palma, M., Peralta, A., Prado M. J. y González M. F. (2002). Estado actual y dinámica de los recursos forestales de México. Biodiversitas, 41, 8-15.

Zapata-Sierra, A. \& Manzano-Agugliaro, F. (2008). Influencia de seis especies arbóreas en la infiltración de agua en el suelo. Agrociencia, 42(7), 835845. Recuperado en http://www.redalyc.org/articulo.oa?id=30211207010.

Zhang, X., Qi, X., Zhou, X., \& Pang, H. (2006). An in situ method to measure the longitudinal and transverse dispersion coefficients of solute transport in soil. Journal of Hydrology, 328(3-4), 614-619. DOI: $10.1016 / j . j h y d r o l .2006 .01 .004$.

Zhang, Z. B., Peng, X., Wang, L. L., Zhao, Q. G., \& Lin, H. (2013). Temporal changes in shrinkage behavior of two paddy soils under alternative flooding and drying cycles and its consequence on percolation. Geoderma, 192, 12-20. Recuperado de http://dx.doi.org/10.1016/j.geoderma.2012.08.009 